\title{
A Parental Perspective of the Screen Devices Influence on Children: Chinese Families' Anxiety Among Entertainment, Education and Social Needs
}

\author{
Junxi $\operatorname{Lin}^{1, * a, \dagger}{ }^{*}$ Jiale $\operatorname{Lin}^{2, b, \dagger}$
}

\author{
${ }^{I}$ Suffolk University, Boston, USA \\ ${ }^{2}$ St. Anne's-Belfield School, Charlottesville, USA

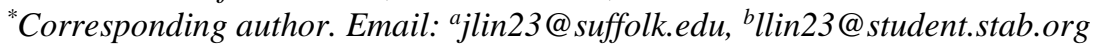

These authors contributed equally.

\begin{abstract}
This article describes the views of parents of Chinese families on their children's use of touch-screen devices in an era of rapid media technology development. This research relates to the new-media influence issue and inter-generational relationship of the Chinese family. It contains multiple dimensional approaches to explore the relationship between parents and children who utilize screen devices at a relatively young age. Our main research methods are questionnaires and semi-structured interviews. The main content comes from a case in the interview, which talks about the experience of a boy named Joey from a high-income family as a video uploader. All cases are collected randomly from welldeveloped cities in southeast China and represent the new generation family mode. Moreover, the evidence in the passage includes practical cases that a kid is supported by his family to express his own opinions about fashion on the Tik Tok and theoretical knowledge. The result of the research can be applied to various situations in the future. One of the most crucial points we got is that the influence of screen devices on kids is diverse, and parents' understanding of their children's social or entertainment needs serves as a more and more essential part most of the time.
\end{abstract}

Keywords: Screen devices, Parental anxiety, Entertainment influence

\section{INTRODUCTION}

As early as the nineteenth century, Alexis De Tocqueville asserted in his book Democracy in America, 'I am most worried about the United States not because it promotes extreme democracy, but because it has too few measures against tyranny' [1]. He has pointed out many times that there is a 'blind instinct' in a modern democracy. That is to say, once the modern democracy that pursues equality loses the guarantee of freedom, it will easily lead to the tyranny of the majority and easily lead to the enslavement of individuals. Individual spiritual freedom causes distortions on two levels. Obviously, Tocqueville has insight into the potential conflict between the modern democratic system and the spirit of freedom. He reminded people that the greatest danger of a democratic system is not anarchy but that its absolute authority stifles individual freedom in the majority's name. In Tocqueville's eyes, citizens receiving civic education and participating in politics are essential to guarantee freedom. Since the beginning of the 21st century, the United States has emphasized civic education under the global era's multicultural background. Starting from the concept of cultivating global citizenship, based on respecting national equality and dignity, it has cultivated qualified citizens who participate in national political, economic, and cultural activities. A popular culture stemming from this globalization progress strengthens global citizens' commonality by diluting differences in culture and identity. But when we look back at the beginning of 2021, ten social media platforms led by Twitter due to the Capitol Hill incident permanently banned the account of former President Trump on the grounds of hate speech and threats of violence [2]. It took decades for people to accept that social media serves as a globalization and culture integration tool. This makes us reflect on whether social media has become a mainstream media consumption tool for younger generations such as Gen $\mathrm{Z}$ or post-Gen Z, whether their views and attitudes towards 
civic education and democratic systems have also been affected.

No matter how it is described in a Machiavellian way, the understanding of Tocqueville on how the majority might manipulate civic education shows just one fold of how today's communication technology brings trouble to parents [3]. On one hand, people worry about their children being poisoned by highly addicted social media behaviour as if parents are no longer with the privilege of being the kid's eloquent and life-time educator. On the other hand company of information technology set the education issue to another level. Screen device producers like Apple company published technical standards to guarantee that activities on touch screen devices like iPad or mobile phones ignite the creativity of children [4]. With the help and prosperity of the mobile application market, screen devices' promotion does good to children's intellect development continues [5-7]. More and more proves to prevail, and parents once reluctant to embrace the children-screen devices parents interaction mode are now convinced for there is no harm for the children's learning performance [8-10]. The approval of having screen devices happens, yet the anxiety of parents remains. This article uses a combination of questionnaires and interviews, trying to use the intergenerational relationship between parents and younger generations as an entry point to explore how social media can blur the user identity between content exporters and passive receivers. The role of today's social media is no longer limited to satisfying users' entertainment and social needs, which further has a more profound impact on civic education.

\section{SOCIAL, ENTERTAINMENT NEEDS AND CIVIC EDUCATION}

Social media and digital media define modern communication as they adopt a many-to-many network communication method. Because of its interpersonal communication interaction, it breaks the limitations of one-to-one and one-to-many in interpersonal communication, thus changing the audience's status has also changed the relationship between the communicator and the communication tool. The primary motivation for the use of social media at home and abroad is to meet social needs. Social needs are the third level of Maslow's needs hierarchy and one of the motivations for using and satisfying earlier studies [11]. In this demand, users can contact old friends or make new friends, and people are eager to interact with like-minded people. Research shows that users accustomed to using social media have higher social needs than users who do not use social media often and thus spend more time on social media and get more satisfaction.

The entertainment need is a bit different from the social one. Social media provides users with a lot of entertainment materials. In the era of generalized entertainment, people tend to read shallowly and think shallowly. Users can easily be attracted by entertainment gossip and pass the time happily. In many studies, seeking entertainment is a common motivation for use and satisfaction. Due to social interaction, social media platforms develop into entertainment centers. Social interaction is no longer a purely "social" activity but a more purposeful activity, especially based on content consumption.

Moreover, one of the negative effects of social media on civic education is the rise of populism and gender antagonisms on social media in recent years. The misogyny culture was certainly not invented by the Internet, but social media has a great opportunity to deliver misogyny culture to young new netizens very efficiently. Online activities are occupying an increasing proportion of people's conceptual life. If in the themes that children like and the communities they identify with, what they see daily is that the KOLs and netizens they cited as comrades all take misogyny for pleasure, feminism as a plague, and split gender opposition as cool things. They certainly have a good chance to think that these are just. After all, this is not the case for one person, but for a group of people, a community that agrees with each other. Then the mainstream order, such as school education, does not have too many things that directly offset the misogynistic culture. How can it be stopped? This, of course, already smells of a culture war. It is extremely difficult for adults with opposing ideas to change their minds. But they still go all out to fight for the right to speak, and a long-term goal is to compete for the next generation. It will be a real game-changer. And now that social media is so convenient that it can easily directly affect new netizens a few years younger than themselves, unlike in the past, they have to slowly climb the social power ladder to gain a voice.

On the one hand, disseminating diverse political information in social media promotes youths to identify with citizenship. In today's society, young people have used social media as an important channel to understand themselves and understand the world, especially to obtain information on major events. In this kind of information channel as a self-media, users can directly produce or freely produce and publish information on mobile phones through third-party software. Traditional information checks and restrictions are almost in vain, and the diversification of information is greatly improved. Under the influence of this diversified information, especially through onlookers or discussions on various major political, economic and social events, most young people gradually recognized the collective identity of citizens and believed that as citizens, they should enjoy the knowledge required by the law. Rights are established and guaranteed, which include expression rights, participation rights, and supervision rights. In the socialization of traditional politics, identity is rapidly being subverted. In the social media environment, individual identity no 
longer needs to be completely restricted by traditional dimensions such as class, status, gender, race, or bureaucratic jurisdiction. Besides, they are not restricted by the traditional forms of mass media. Teenagers who were originally not interested in politics have obtained unprecedented rights of expression and participation through social media, have direct access to various dialogues, and form insights through the real world's multi-party interaction and the virtual world. In this way, the citizenship identity in the political system and the legal context is born through information dissemination and interactive communication in social media.

On the other hand, social media promotes the true construction of the public sphere and provides space for identifying and constructing youth citizenship. Since the public sphere is a place for citizens between the state and society to participate in public affairs, it is located outside of political power. It is an independent space relative to the private sphere. In this space, citizens with rational and critical consciousness are not subject to power institutions' interference and can openly debate and criticize public affairs freely. Although newspapers, radio, and television have a certain influence and effect on the public sphere in the era of traditional media, the public sphere is often just a utopia. The so-called public sphere is only a space that is distorted and used by power groups and elites to a considerable extent. Due to the existence of leading factors such as agenda-setting, media filtering, and various independent opinion leaders, the voices of the grassroots are either obscured or suppressed or assimilated. Even if they are listened to, it is the result of the selection of the elite. In this way, a self-formed public sphere characterized by criticism has gradually reduced to an institutional, institutional and manipulative "pseudo-public sphere". The rapid development of network information technology has given the grassroots a platform to make their voices heard, but only with the emergence of social media can grassroots opinions have the opportunity to be truly expressed and be widely disseminated. In terms of its communication characteristics, social media is the closest channel to the public sphere's political goal under current technology and social conditions. It has a direct impact on the true construction of the public sphere. In the era of new media led by digital media, young people can discuss and make judgments on public policies, political events, and other issues of common concern in this space so that the participating young people can quickly form a citizenship identity. To their parents' concern, this process is for most of the occasional lack of older generations' participation.

In modern society, there is a trend that children rely more on screen devices, such as mobile phones, computers, and iPad. Diverse different categories of apps are created by people, which are appealing to the young. Due to the young ages of kids, parents will consider that their children always play the role of receivers in this circumstance. Namely, children just receive all of the information from the internet without filtering. Gradually, such a misunderstanding root in people's minds for a long period. However, society's unprecedented development forces more children to express and output their thoughts in diverse ways. This phenomenon can be explained by both the advanced technology nowadays and the precocious minds of kids. Therefore, there are two models in the relationship between teenagers and screen devices' utilization, which contains a positive producer and a negative taker. Obviously, positive producers appear more frequently in modern society.

Two classic platforms in China for individuals to convey ideas are Tik Tok and BiliBili. The former is a short video app, and the latter is more diverse. Since the limitation for teenagers using these apps is not strict rather than the entertainment industry, it is facilitated to access and share information with strangers. In a sense, several laws prohibit any underage from participating in commercial performances or commercial advertisements. One of the prominent reasons to set laws is to protect children's development. Nevertheless, restriction on the internet is quite less, which assists nonage to convey their experience. Take Tik Tok, a typical and popular short video platform, as an example. The use of Tik Tok is particularly simple that children only have to record a video, edit it and then upload it. Kids can upload whatever things that they are interested in legally. According to the collected data, more than $40 \%$ of underage boys share their favourite sneakers and fashion brands; $60 \%$ of underage girls share their makeup and clothes. Also, a bunch of children is more likely to record their daily lives directly by Vlog. Data shows that the frequency and number of juveniles using Tik Tok are increasing in a forward slope.

\section{METHOD}

We interviewed 5 parents about their children's situation about using electronic devices, and the purpose of interviewing is to discover deeper effects on kids. These families were selected randomly while represented some basic attributes of the traditional Chinese style education environment. It was worthy to notice that one of their kids was gaining knowledge abroad, and the other stayed in China. It did not mean that the family educated them in the Eastern way. In fact, parents were more sensitive about modern socialization, and most of them even supported their kids to use screen devices to express ideas adequately. We prepared eight interview questions: when did you let your children touch the following electronic products? Why did you want them to have access to these electronic devices? Have you discovered the impact of these electronic devices on children? What does your child do with these electronic products? Do you think utilize electronic products is helpful to the formation of children's interest? What do you think is the worst 
impact on children after giving them electronic products? Will children use electronic products more frequently during the quarantine? What is your attitude towards these electronic products/has it changed? The interview's feedback was extremely inductive for our research: The biggest negative effect of using electronic products was damaging to the health of children, including their eyesight and cranial nerve. Also, parents stated that children began dependent on electronic devices and could not leave with them. It was more and more laborious for kids to manage their time productively by themselves, which made parents anxious. Honestly, parents would ignore the effects of screen devices on their kids due to the generation gap. In conclusion, we designed to use the interview as a probe to investigate whether parents were aware of modern touchscreen devices' influence on their kids' behaviour. Especially, the sixth question listed above would be a topic developed in the future passage since we were able to figure out that parents did not understand the impacts of advanced screen devices deeply.

\section{A CASE STUDY OF THE POSITIVE MODE: JOEY AND HIS PARENTS}

A kid of one of my interviewers has the experience of uploading short videos to Tik Tok. His name is Joey, and he is in the seventh grade now. Until now, he has more than two thousand followers on the platform. Most of his videos talk about fashion sneakers, like exclusive Air Jordan, limited collaboration shoes, and so on. Every time, Joey will introduce his new shoes, including their background information, designer, and other related stories. Through his videos, people can easily figure out that he is really interested in this area. However, due to his age, there are a bunch of more "important" tasks he has to do, such as his academic performance in school. Gradually, the update frequency is lower and even stops update these days.

It is reasonable for parents to generate an anxious mood regarding their children's use of mobile phones for parents. Due to the unprecedented development of technology, most people cannot catch up with the new trend of society nowadays. Therefore, their value is generally backward, explaining why they don't approve of their children playing apps like Tik Tok on mobile phones. There are two kinds of worries of parents. The anxiety of the recipient's parents may be relatively single compared with the producer's parents. Recipients' parents may only concern about children's physical health, like deterioration of eyesight. Furthermore, parents are more willing to let their kids pay more attention to the academy. Nevertheless, the producer's parents will have much diversified worries due to several factors. Firstly, adolescents are not mature enough to suffer aggressive comments from those virtual friends. Some adults will turn the misfortunes they have encountered in life into negative energy and then vent them through Tik Tok's platform. In this circumstance, many innocent teenagers will be hurt by those words, and this kind of spiritual hurt is hard to make up in the future. This will even leave them a long-last shadow. Parents who support their children uploading videos is likely to feel self-condemned and repentant. They consider that if they do not encourage their children to do such an activity, their children are able to avoid accepting malicious speech from others. Secondly, there are many seductive and deceptive posts on Tik Tok, such as deceiving teenagers to sign companies that will earn countless money. Because of Tik Tok uploaders' younger age, many fraud companies take this opportunity to make money. The last one is the most common that parents will worry that their children's academic performance will be affected by posting videos on Tik Tok. Though children's grades are still constantly good, they also have a prejudice against playing mobile phones that there must be negative impacts. Joey, a kid in my interview, suffered cyber violence when he was in elementary school. He likes to share his new shoes with strangers, and he will post every relative information about that shoes clearly every time. It stands to reason that people should admire him very much because he started making videos at such a young age and is willing to share with others. Ironically, he failed to receive the praise he deserved, but he received sarcasm from others instead. This is very unfair to him because those strangers are jealous that Joey has a lot of limited shoes. Due to those shoes' high expenses, they couldn't get them, so they begin to accuse Joey of buying fakes and "kindly" let him learn useful knowledge. Their comments hurt Joey very much since Joey has never been hurt like this by others. His age is so young that he shouldn't and can't bear the constant verbal attacks from the outside world. Meanwhile, after his parents learn of this, their attitude towards the use of Tik Tok changed. They go from the original unconditional support to the current intervention management to protect their son's mental health. Fortunately, Joey has a powerful mental state, so he is still very optimistic nowadays. The case of Joey and his family shows that even if parents have no concerns in the process that their children being through the evolution of new era social, entertainment, and civic education mode, a positive engagement for the whole family still works.

\section{CONCLUSION}

According to the material, analysis, and detailed description mentioned above, we investigate a case of positive engagement of screen devices based on intergenerational family relationships. The research's kernel is the attitude of parents to their kids about using screen devices in an era with pervasive and highly developed technology. After studying related material for a while, one of the most widespread phenomena displays that it is somewhat arduous for parents to accept screen 
devices' influence, like change in the value, on their children. Otherwise, some parents consider those apps a way to express thoughts to the public and support their kids to do such things. Indeed, social mobile apps will bring both positive and negative impacts, which emphasizes parents' significance. Under such a circumstance, the belief of parents plays a more decisive role in children's effects. The target should be concerned is not only limited to people who use screen devices. We also have to care about the relationship between people who have been on standby. In the future, it is quite interesting to focus on more problems brought by utilizing screen devices.

\section{REFERENCES}

[1] Tocqueville, Alexis de. Democracy in America, Chicago: University of Chicago, 2000

[2] A permanent suspension of social media account of Donald Trump, retrieved from https://blog.twitter.com/en_us/topics/company/202 0/suspension.html

[3] Harvey C. Mansfield \& Delba Winthrop, Tocqueville's Machiavellianism, Perspectives on Political Science, 43:2, 2014, pp. 87-92,

[4] Apple (2017). iPad in Education. Available inline at: https://images. apple.com/education/docs/ipadin- education- results.pdf (Accessed March 30, 2018)

[5] Baccaglini-Frank, A., and Maracci, M. (2015). Multi-touch technology and preschoolers' development of number-sense. Digital Exp. Math. Educ. 1, 7-27

[6] Beach, R., and Castek, J. (2015). "Use of apps and devices for fostering mobile learning of literacy practices," in Handbook of Research on the Societal Impact of Digital Media, eds B. Guzzetti and M. Lesley (Hershey, PA: IGI Publishers), 343-370.

[7] Bebell, D., and Pedulla, J. (2015). A quantitative investigation into the impacts of $1: 1$ iPads on early learners' ELA and math achievement. J. Inf. Technol. Educ. 14, 191-215.

[8] Berney, S., and Bétrancourt, M. (2016). Does animation enhance learning? A meta- analysis. Comput. Educ. 101, 150-167.

[9] Becker, D. R., McClelland, M. M., Loprinzi, P., and Trost, S. G. (2014). Physical activity, selfregulation, and early academic achievement in preschool children. Early Educ. Dev. 25, 56-70.

[10] Boncoddo, R., Dixon, J. A., and Kelley, E. (2010). The emergence of a novel representation from action: Evidence from preschoolers. Dev. Sci. 13, 370-377.

[11] Examining Maslow's Hierarchy Need Theory in the Social Media Adoption, Sanchita Ghatak, Surabhi Singh, FIIB Business Review, vol. 8, 4: 2019, pp. 292-302. 